\title{
Efectos del Innovation Project-Based Learning en la Formación de Educadores Kinesiólogos en Innovación
}

\author{
Effects of the Innovation Project-Based Learning in the Training of Kinesiologist \\ Educators in Innovation
}

Jorge Ignacio Maluenda Albornoz ${ }^{1}$

Recibido: 17-10-2020; Aceptado: 19-01-2021; Publicado: 20-01-2021

\section{RESUMEN}

Objetivo: Evaluar los efectos de la implementación de la estrategia metodológica "Innovation Project Based-Leargning" sobre el desarrollo de habilidades para la enseñanza de la Innovación. Método: Se utiliza un diseño pre-experimental con pre-test y pos-test en un grupo de 11 Docentes de Kinesiología. La intervención consiste en el uso de la metodología en el contexto de un Diploma para la Formación de Formadores en Innovación. Se utiliza un instrumento estandarizado para medir la habilidad para Innovar, y preguntas adicionales para captar la percepción de los participantes sobre el efecto de la estrategia en su habilidad docente. Resultados: Se observó un aumento de los puntajes post-test en todas las dimensiones evaluadas tanto del instrumento estandarizado, como de las preguntas sobre la percepción del efecto de la estrategia. La estrategia muestra un efecto sobre las 5 habilidades consideradas y sobre la percepción de los Docentes para la enseñanza de la Innovación. Conclusiones: Se aprecia nueva evidencia a favor de la estrategia para desarrollar competencia en Innovación.

Palabras clave: Innovation Project-based Learning; Aprendizaje basado en Proyectos; Proyectos; Innovación; Enseñanza; Aprendizaje; Formación de Formadores; Educación Superior; Perfeccionamiento Docente; Innovación Docente; Aprendizaje Colaborativo.

\section{ABSTRACT}

Objective: To evaluate the effects of the implementation of the methodological strategy "Innovation Project Based-Learning" on the development of skills for teaching Innovation. Method: A preexperimental design with pre-test and post-test is used in a group of 11 Kinesiology Teachers. The intervention consists of the use of the methodology in the context of a Diploma for the Training of Trainers in Innovation. A standardized instrument is used to measure the ability to innovate, and additional questions are used to capture the perception of the participants about the effect of the strategy on their teaching ability. Results: An increase in post-test scores was observed in all the dimensions evaluated, both standardized instrument, and of the questions about the 
perception of the effect of the strategy. The strategy shows an effect on the 5 skills considered and on the perception of Teachers for the teaching of Innovation. Conclusions: New evidence is appreciated in favor of the strategy to develop competence in Innovation.

Keywords: Innovation Project-based Learning; Project-based learning; Projects; Innovation; Teaching; Learning; Training of trainers; Higher education; Teaching improvement; Teaching Innovation; Collaborative learning.

\section{INTRODUCCIÓN}

La sociedad del siglo XXI está en constante cambio, sujeta a nuevas y variadas demandas que requieren ser atendidas. Por esta razón, se requiere que los Profesionales desarrollen competencias para enfrentar la complejidad y dinamismo de los problemas, con flexibilidad, rigurosidad y eficiencia. Se hace cada vez más relevante la formación de Técnicos y Profesionales en base a competencias genéricas: competencias que permiten desempeñarse en distintos contextos, con independencia de la profesión estudiada (Hernández, Alvarado y Luna, 2020), además de permitir una actualización permanente y el fortalecimiento de las capacidades necesarias para seguir aprendiendo a lo largo de la vida (Navarro et al., 2015).

Entre las competencias más necesarias se encuentra la capacidad para Innovar. La Innovación, entendida como un fenómeno intelectual, es la acción de dar origen a un nuevo elemento, ya sea mediante la transformación de uno preexistente o la concreción de una nueva idea con la introducción de alguna novedad (Sebastian, 2010). En el contexto económico, implica además que la introducción de la novedad genere valor económico.

Desde la perspectiva de este autor, la Innovación es el proceso a través del cual se genera una solución novedosa que agrega valor al aportar a la satisfacción de una necesidad social, en cuyo desarrollo participan distintos actores sociales. Cuando se cumplen estas condiciones, decanta no solo en la generación de soluciones que agregan valor, sino que también en el fortalecimiento de las capacidades sociales para la elaboración posterior de soluciones futuras.

En el contexto educativo, desarrollar competencias para innovar permite a los Estudiantes y futuros Profesionales enfrentar desafíos en su quehacer cotidiano con respuestas alternativas, que busquen mayor eficiencia y contemplen sus efectos en el entorno (Maluenda y Lledó, 2019; Tarango, Guajardo-Morales, Machin-Mastromatteo y Villanueva-Ledezma, 2020; Granados, Vargas y Vargas, 2020). Con esta habilidad, son capaces de crear, generar, transformar y adaptarse a cada nuevo contexto con múltiples soluciones novedosas y eficaces mediante un pensamiento analítico, sistémico y crítico (Hernández, Alvarado y Luna, 2020).

Formar personas que se incorporan a las organizaciones públicas y privadas, que cuentan con la capacidad de innovar y emprender proyectos o acciones que mejoren necesidades o problemas vigentes, permite agregar valor a los procesos. La inversión en el desarrollo de competencias e Innovación en pequeñas y medianas Empresas, por ejemplo, ha demostrado ser un factor que agrega valor en los comportamientos organizacionales y los productos que se alcanzan, así como los resultados que obtienen en sus actividades industriales (Pastor, 2017).

En la actualidad, el desarrollo de habilidades para innovar se realiza en distintas modalidades. En algunos casos, se realiza a través de la enseñanza tradicional teórico-práctica en Instituciones de Educación Superior en el Pregrado y Posgrado, o a través de capacitación y asistencia técnica a Trabajadores y Empresas que requieren de dichas capacidades. Otro enfoque ha sido el apoyo de pequeñas, medianas y grandes Empresas que requieren de estas capacidades, a través de la mentoría, la incubación y aceleración de su desarrollo como organizaciones.

En los últimos años, se han puesto a prueba numerosas experiencias en Pregrado basadas en proyectos para desarrollar competencias en Innovación. Los Estudiantes obtienen buenos resultados en el logro de proyectos estudiantiles, con carácter Innovador y mayores aprendizajes en los Estudiantes (Arias-Flores, Jadán-Guerrero y Gómez-Luna, 2019; Badia y Martínez, 2017; 
Cuevas, Rivera, y Pardo, 2017; Bisang y Fuchs, 2017). El eje común de estas estrategias es el uso de un problema o necesidad para estimular el trabajo de los Estudiantes en la oferta de soluciones pertinentes a dicho problema y el desarrollo de actividades conjuntas para alimentar el proceso a través del análisis y la opinión externa. Sin embargo, en general, carecen de un modelo pedagógico de base o de claras directrices para estimular intencionadamente el aprendizaje. Esto, porque el foco principal es abordar el trabajo de aprendizaje como si fuera un proyecto de Innovación real, incluso a veces perdiendo el foco de la actividad educativa. De este modo se observa que no se han diseñado específicamente para fines educativos, sino que, usualmente, para desarrollar innovaciones, aspecto problemático si consideramos que el éxito en el aprendizaje está dado por el diseño, aplicación y evaluación de las herramientas apropiadas para la competencia (Arias, Giraldo, y Anaya, 2013).

Por otra parte, la formación de Innovadores ha sido más pobremente abordada en educación superior en Iberoamérica aún. La formación en esta área es muy escasa y se limita a la enseñanza de conceptos, métodos y claves para innovar y emprender, pero no al "cómo desarrollar" competencias en Innovación y emprendimiento en otros. Esto, ha llevado como consecuencia dificultades en la transferencia de conocimiento a las aplicaciones reales (Cruz, 2016). En parte porque existen escasos Formadores capacitados para realizar la labor de educar las futuras generaciones en estos temas $y$, por otra parte, por los escasos programas que hayan sido diseñados específicamente para formar Formadores de la Innovación (Tarango, GuajardoMorales, Machin-Mastromatteo y Villanueva-Ledezma, 2020; Granados, Vargas y Vargas, 2020).

El (i)PBL (Innovation Project Based Learning) es una estrategia metodológica diseñada específicamente para la enseñanza-aprendizaje de la Innovación, propuesta por el autor del presente artículo en el año 2018, que ha sido probada en algunas experiencias de enseñanza con Estudiantes de Pregrado de Kinesiología (Maluenda y Dubó, 2018) y de ingeniería (Maluenda, Lledó y Uribe, 2019) mostrando un impacto sobre las 5 habilidades consideradas fundamentales en personas que innovan y/o emprenden (Dyer, Gregersen y Christensen, 2009). Los efectos encontrados en estas intervenciones han mostrado magnitudes del efecto Moderadas a Altas, y han sido igualmente relevantes para Estudiantes de distintas disciplinas (Maluenda y Dubó, 2018; Maluenda, Lledó y Uribe, 2019). En estas investigaciones, además, se ha observado que la estrategia tiene un efecto moderado sobre la autoeficacia de los Estudiantes para innovar y aumentar su disposición a involucrarse en iniciativas personales y colectivas con dichos fines.

El objetivo de la presente Investigación fue evaluar el efecto del (i)PBL en el desarrollo de habilidades necesarias para la enseñanza de la Innovación en un grupo de Docentes de Kinesiología. Además, se buscó evaluar la percepción de estos Docentes frente a su capacidad para enseñar la Innovación.

\section{MÉTODO}

Participantes. Muestra de 11 Docentes de la carrera de Kinesiología de la Universidad de Concepción, el 63,63\% hombres y el 36,36\% mujeres, con edades entre 30 y 45 años. Este grupo de Docentes participó de manera voluntaria en el curso de formación titulado "Diploma Formadores en Innovación" diseñado en base a la estrategia (i)PBL. Su participación en el programa de formación y en el estudio fue voluntaria.

Diseño de Investigación. La Investigación corresponde a un diseño pre-experimental con pretest y pos-test. Se conformó un solo grupo con los participantes. Estos fueron evaluados antes y después de la intervención con un instrumento estandarizado. Adicionalmente, se realizaron 3 preguntas para evaluar la percepción de los Docentes sobre la formación. Se utilizó el análisis de las diferencias entre las medias a partir de la Prueba T de Student, previo al testeo y cumplimiento de los supuestos para esta prueba. Los análisis se realizaron a partir del software estadísticos SPSS Versión 22.

Instrumento. El instrumento utilizado es el Innovator's Behavior Questionnaire - (i)BQ (Maluenda y Lledó, 2019). Este instrumento evalúa el desarrollo de 5 habilidades (observación, 
reflexión, networking, experimentación y asociación conceptual) consideradas indispensables para Innovar (Maluenda, Lledó y Uribe, 2019).

Este instrumento fue diseñado con base en las 5 microcompentencias o habilidades necesarias para que una persona sea un Innovador competente propuestas por Dyer, Gregersen y Christensen (2009). Así, la competencia en Innovación es entendida como un conjunto de habilidades que, en interacción, favorecen la capacidad de un individuo para enfrentar desafíos y problemas con una mirada innovadora. Estas habilidades son:

Asociación Conceptual. Entendida como la capacidad para "conectar los puntos", realizar conexiones inesperadas, combinar las piezas de información de forma que se produce una nueva idea innovadora.

Observación. Habilidad que destaca el carácter meticuloso y ocurre cuando las personas son capaces de detectar aspectos que no son evidentes o de fácil percepción.

Cuestionamiento/reflexión: Es entendido como la capacidad para realizar las preguntas apropiadas a las observaciones realizadas. En este sentido se convierte en una herramienta de análisis crítico que permite someter a prueba el status quo y encontrar brechas, alternativas y mejoras potenciales.

Experimentación. Se refiere a una forma de afrontamiento de los desafíos que implica la prueba y testeo de variadas potenciales soluciones frente a una problemática permitiendo no solo permite la generación de las soluciones finales, mediante un refinamiento progresivo, sino también construir las capacidades que serán clave para el éxito de los proyectos en el futuro.

Trabajo en red (Networking). Se refiere a la forma de abordar los desafíos que se vale de las miradas de diversas personas, muchas opuestas y/o críticas, que permitan desafiar seriamente la propia en la búsqueda de mejores soluciones. Desde la perspectiva de Maluenda y Lledó, (2019) es, además, una forma de crear tejido social que permita el desarrollo de los proyectos emprendidos y de transferir capacidades a las comunidades y actores involucrados.

El estudio de validación del instrumento mostró excelentes propiedades psicométricas a partir del análisis de la validez de constructo mediante Análisis Factorial Confirmatorio (RMSEA = 0.05, 95 $\%$ CI $[0.021,0.072]$, CFI $=.944$ y TLI $=.932)$ y el análisis de la confiabilidad en la escala global $(a=0.905 ; \omega=0.907)$ en base a los estándares recomendados por la literatura (Hair et al., 2014).

A este instrumento se le agregó una pregunta orientada a captar cambios en el sentido de autoeficacia para innovar de los participantes: ¿Qué tan capaz te sientes de iniciar una Innovación? Adicionalmente, se utilizaron 3 preguntas para pesquisar la percepción de los Docentes sobre el impacto de la estrategia en su comportamiento: a) ¿Cuánto aportó la estrategia al desarrollo de mis habilidades para le enseñanza de la Innovación?; b) ¿En qué grado aportó la estrategia a mi dominio como docente?; c) ¿Qué tan probable es que yo implemente esta estrategia con mis Estudiantes el siguiente semestre? Todos los instrumentos tuvieron una escala de respuesta de 1 a 7, donde 1 significó el mínimo valor y 7 el máximo valor en cada ítem.

Por último, a modo de seguimiento, se consultó a los Docentes vía correo electrónico o conversación telefónica si habían implementado la estrategia metodológica diseñada durante el Diploma en alguna de sus asignaturas. Este seguimiento, se realizó a 3 meses de finalizado el curso.

El Innovation Project Based Learning - (I)PBL. La estrategia (i)PBL utiliza como eje vertebral la elaboración de un proyecto a partir del planteamiento de un desafío de Innovación. Desde este desafío, los participantes deben elaborar soluciones siguiendo un proceso de fases iterativas tipo "sprint de Innovación" bien definido: problematización, ideación, prototipado y testeo. Este sprint se puede repetir hasta alcanzar los objetivos pedagógicos en cuestión, o, eventualmente, en el concurso de un desafío de Innovación real, hasta alcanzar un producto mínimo viable. 
La base pedagógica de la metodología requiere la incorporación de tres principios:

a) La experimentación, que implica que los Estudiantes encarnan activamente el proceso de Innovación frente a un desafío real o hipotético. Se sustenta en el enfoque de aprendizaje activoparticipativo y busca que los Estudiantes aprendan sobre el proceso y encarnen los efectos de los resultados obtenidos (Bonwell y Eison, 1991).

b) La colaboración, que implica la interacción permanente con los usuarios o potenciales usuarios, pares, especialistas, organizaciones civiles e instituciones públicas vinculadas donde lo medular es el intercambio permanente con otras miradas que permitan enriquecer las soluciones y favorecer un máximo ajuste a las necesidades de los usuarios finales. Este componente se sustenta en la perspectiva del aprendizaje colaborativo que entiende el aprendizaje como proceso social solo a través del cual, se desarrollan capacidades indispensables como la efectividad comunicativa, la negociación y transacción además del ejercicio de componentes asociados a la inteligencia interpersonal (Johnson, Johnson y Holubec, 1999; González, Maluenda y Navarro, 2015).

c) El uso de herramientas para favorecer procesos metacognitivos a nivel individual y colectivo (equipo) de modo que logren ser competentes en Innovación. Este componente es crucial puesto que busca que los Estudiantes aprendan sobre las condiciones de mejor funcionamiento del equipo, las capacidades individuales y los mejores mindset personales para poner las potencialidades de cada miembro al servicio de los objetivos colectivos. Este componente se sustenta en el enfoque de autorregulación del aprendizaje que plantea como un componente indispensable del aprender la planificación, ejecución y evaluación de las propias actividades (Panadero, 2017; Zimmerman y Labuhn, 2012).

La estrategia (i)PBL establece un curso de acción sugerido y principios educativos flexibles que deben orientar el desarrollo del proyecto de clases. De esta forma, se busca generar el desarrollo de competencias de forma intencionada, planificada y verificadas.

Para la presente Investigación se utilizó como estrategia de enseñanza-aprendizaje el (i)PBL siguiendo las recomendaciones planteadas en "Innovation Project Based Learning. El método y directrices para su implementación" (Maluenda, 2019).

El curso (Diploma) tuvo una duración de 3 meses, con un total de 12 sesiones de trabajo (una por semana), una carga de 3 horas cronológicas semanales presenciales y 3 horas cronológicas de trabajo autónomo. Cada sesión contempló el trabajo de 1,5 horas teóricas y 1,5 horas en el avance de proyecto. El trabajo autónomo fue dedicado a la reflexión sobre los aprendizajes propios, la evolución del proyecto y el trabajo del equipo, apoyada por preguntas y guía de los Docentes del Diploma con una duración aproximada de 3 horas cronológicas a la semana. Adicionalmente, los Docentes realizaron actividades de validación del problema, las potenciales ideas de solución y los diseños de la solución. Este se desarrolló durante el segundo semestre del año 2019. De este modo, la forma de trabajo se describe en la Figura 1.

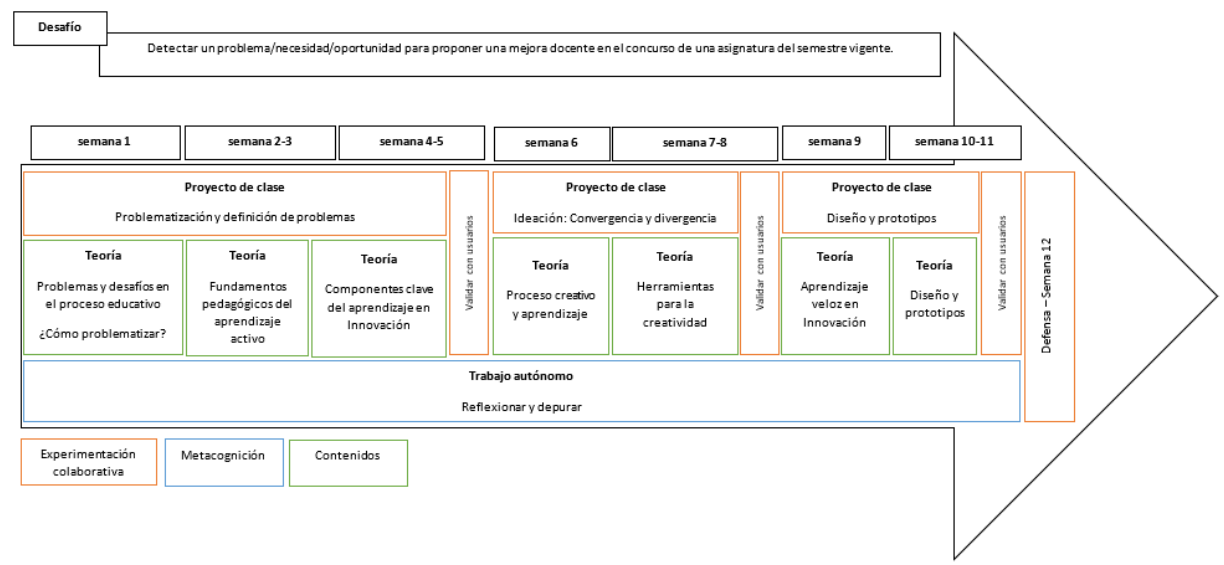

Figura 1. Organización esquemática de la implementación del (i)PBL en la formación de Docentes en i+e 


\section{RESULTADOS}

Los resultados mostraron diferencias estadísticamente significativas entre el pre-test y posttest $(p<0.01)$ realizado a los participantes, reflejando un aumento en el puntaje de la segunda evaluación en todas las mediciones pesquisadas. El aumento más pequeño fue en la dimensión "redes" (Networking) mientras que, el aumento más sustantivo se observó en la dimensión "asociación conceptual" (Figura 2).

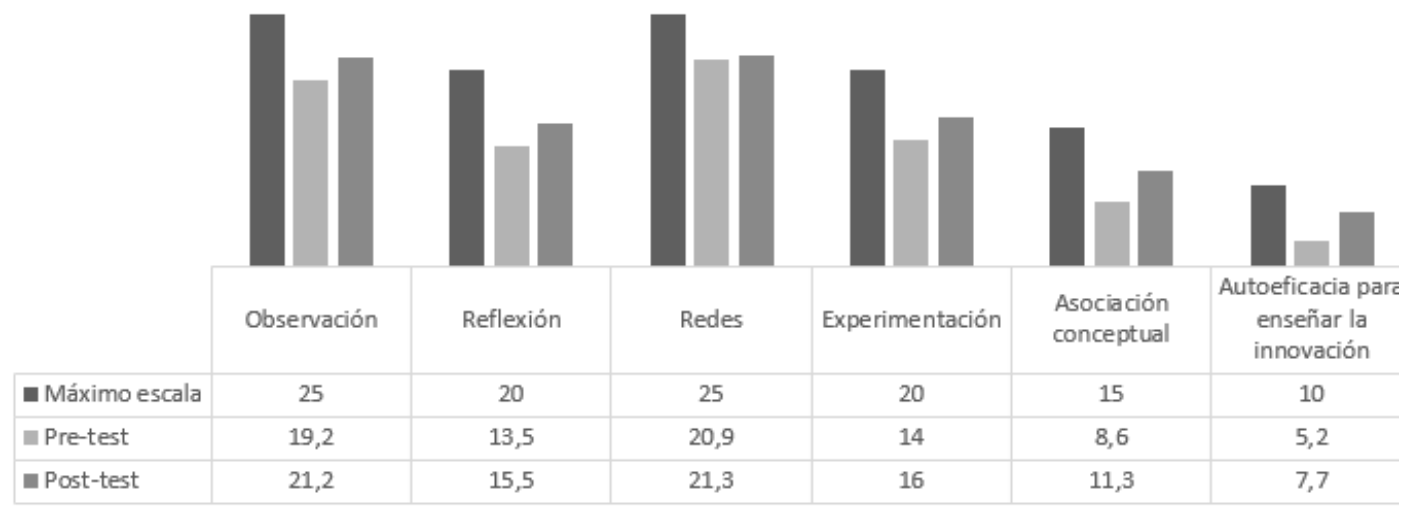

Figura 2. Diferencia en puntajes promedio por dimensión entre evaluación pre y post-test.

Se observó un efecto importante de la estrategia metodológica en las habilidades percibidas por los Docentes para el desarrollo de Proyectos de Innovación.

Por otro lado, en cuanto al dominio pedagógico, se aprecia un aumento del sentido de autoeficacia de los Docentes para la enseñanza de la Innovación. Los Docentes reportaron una contribución sustantiva del (i)PBL para la enseñanza de i+e y de su dominio docente, además de una alta probabilidad de iniciar la enseñanza de la temática en el futuro inmediato (Figura 3).

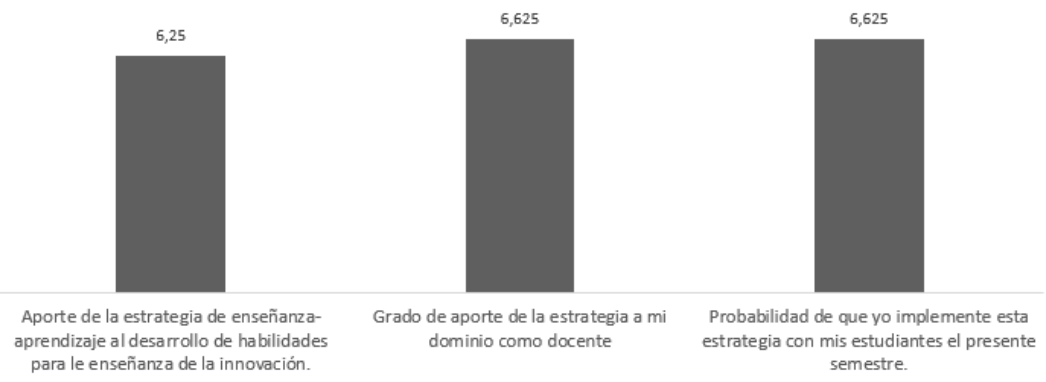

Figura 3. Percepción docente del impacto de la estrategia.

Por último, en una medida de seguimiento, se consultó a los Docentes sobre la implementación en sus clases de la estrategia para contrastar con la probabilidad reportada por ellos mismos al final del Diploma. Nueve Docentes del total implementaron la estrategia en el semestre consecutivo, mostrando una alta consistencia con la estimación realizada por ellos mismos. En todos los casos, se implementó la mejora docente diseñada durante el curso en su completitud.

\section{DISCUSIÓN Y CONCLUSIONES}

A partir de los resultados obtenidos es posible observar un efecto de la estrategia metodológica al desarrollo de habilidades necesarias para involucrarse para la Innovación ( 5 habilidades de Dyer) aumentando todos los puntajes en el post-test. Si bien, el aumento de los puntajes fue pequeño en términos absolutos se observaron diferencias estadísticamente significativas entre el 
pre y post test. Además, es relevante considerar que el nivel inicial de los participantes es alto en comparación con el puntaje global de cada dimensión, aspecto que puede incidir en el aumento moderado de los puntajes en el post-test.

Por otra parte, es clave considerar que frente a un período breve de intervención (4 meses con una sesión semanal de 3 horas y un trabajo promedio fuera de aula de 3 horas semanales) un cambio en todas las dimensiones consideradas es un efecto relevante.

También es relevante considerar que la mejora se observó de manera similar en todas las dimensiones evaluadas, lo que refleja un balance de la estrategia para impactar en todos los aspectos incorporados. Todos estos resultados son coherentes con experiencias previas de la aplicación del (i)PBL en Estudiantes de distintas disciplinas donde se ha observado un efecto homogéneo en las distintas habilidades de los Estudiantes para innovar y emprender (Maluenda y Dubó, 2018; Maluenda, Lledó y Uribe, 2019).

En cuanto al dominio pedagógico, la mejora en la propia capacidad percibida para desarrollar la docencia en Innovación (autoeficacia), refleja una disposición psicológica diferente frente al abordaje inicial lo que puede tener un impacto sustantivo en el ejercicio de la enseñanza. Se ha observado en diversa Investigación el efecto de una mayor autoeficacia docente sobre un mejor ejercicio efectivo de la docencia (Vizcaino, López y Kilmenko, 2018; Hernández y Ceniceros, 2018; Díaz y Pérez, 2013).

Es posible que el cambio en la autoeficacia percibida para enseñar esté relacionado, por un lado, con el aprendizaje obtenido en torno al dominio pedagógico, pero también, respecto del dominio y experiencia adquirido en el ejercicio de innovar. En otras palabras, aprender a enseñar la i+e y experimentar el trayecto necesario para generar proyectos de esta naturaleza tiene un efecto sustantivo sobre la propia capacidad percibida para guiar a otros en este ejercicio.

Los resultados observados en las preguntas de percepción complementan la mirada sobre el efecto en lo propiamente pedagógico. Los Docentes perciben un efecto de la estrategia sobre sus habilidades Docentes y consideran la estrategia útil para desarrollar estos aprendizajes.

Tal vez, el resultado más interesante de esta percepción sea la probabilidad estimada de la implementación de la estrategia en sus asignaturas y el contraste con la implementación real de la misma. Se observó que los 11 Docentes iniciales manifestaron una alta probabilidad de implementar los desarrollos efectuados durante el curso en el futuro. Luego, se observó que 9 de ellos implementaron la estrategia en el seguimiento a 3 meses (semestre siguiente al término del Diploma). Estos resultados muestran un efecto adicional que no fue medido de forma directa sobre la motivación de los Docentes para implementar sus propios desarrollos. Es posible que, la combinación de los nuevos aprendizajes, un mayor sentido de autoeficacia en la temática, la experimentación personal de las bondades de este tipo de enseñanza, además de contar con un producto articulado para su ejecución al finalizar el Diploma, produjeran un estímulo adicional a su implementación real.

Una limitación de los presentes resultados es el tamaño de la muestra en el diseño utilizado puesto que, reduce las posibilidades de generalización. La complejidad de contar con Docentes que acepten participar en estudios que involucran su tiempo y dedicación, además de una observación externa de sus prácticas, dificulta la obtención de muestras más amplias. Desde aquí se desprende que, hacia el futuro, se deba utilizar muestras más amplias para probar la replicabilidad de estos hallazgos.

Otra limitación, también asociada a la muestra es que los participantes han accedido al trabajo de aprendizaje y la Investigación propiamente tal de manera voluntaria. Esto restringe los alcances hacia profesores interesados y motivados inicialmente por acceder a formaciones de estas características lo que desafía al futuro, a contar con Docentes menos convencidos y reticentes a este trabajo para evaluar los efectos de la estrategia. 
Una tercera línea futura de estudio se relaciona con el seguimiento de los profesores y el efecto de las estrategias implementadas. En este estudio, se alcanza a observar un efecto de la estrategia en la implementación efectiva de los Docentes de sus mejoras educativas en el semestre contiguo, con una distancia de 3 meses. Sin embargo, sería relevante evaluar si los Docentes persisten en el uso de los aprendizajes en un período más extenso, además de observar si estas implementaciones son de buena calidad y/o exitosas con sus propios Estudiantes.

\section{Financiación}

La presente Investigación recibió el apoyo del Departamento de Kinesiología de la Universidad de Concepción, Chile.

\section{Conflicto de intereses}

El autor declara que no tiene ningún conflicto de interés.

\section{REFERENCIAS}

Arias, C.; Giraldo, D.; Anaya, L. (2013). Competencia creatividad e Innovación: Conceptualización y abordaje en educación. Katharsis, 15, 195-213.

Arias-Flores, H.; Jadán-Guerrero, J.; Gómez-Luna, L. (2019). Innovación educativa en el aula mediante design thinking y game thinking. Hamut'ay, 6 (1), 82-95. http://dx.doi. org/10.21503/hamu.v6i1.1576

Badia, J.; Martínez, V. (2017). Creative Project-based learning to boost technology innovation. Revista D'Innovació Educativa, (18), 1-13. Https://doi.org/10.7203/attic.18.9019

Bisang, R.; Fuchs, M. (2017). El arte de aprender a innovar en un mundo globalizado y cambiante. Santiago de Chile: CAF, CIEPLAN. http://scioteca.caf.com/handle/123456789/1072

Bonwell, C. C.; Eison, J. A. (1991). Active learning: creating excitement in the classroom. Washington, DC: ASHE-ERIC Higher Education Report no. 1.

Cruz, A. (2016). Innovación de base científica-tecnológica desde la universidad de Iberoamérica. Journal of Technological Management Innovation, 11 (4), 1-4.

Cuevas, C., Rivera, D., y Pardo, R. (2017). Lions up: Una experiencia de aprendizaje colaborativo de Innovación que fomenta la interdisciplina en la universidad de Santiago de Chile. In XXX Congreso SOCHEDI 2017. El desafío de la interdisciplinariedad en la Ingeniería y su impacto en la formación profesional. Santiago: SOCHEDI.

Díaz, A.; Pérez, M. (2013). Autoeficacia, enfoque de aprendizaje profundo y estrategias de aprendizaje. International Journal of Developmental and Educational Psychology, 2(1), 341346.

Dyer, J.; Gregersen, H.; Christensen, C. (2009). The Innovator's DNA. Boston: Harvard Business Review.

González, M.; Maluenda, J.; Navarro, G. (2015). Experiencias de la formación de competencias genéricas en educación superior: Casos basados en el conocimiento construido en la Universidad de Concepción, Chile. Universidad de Concepción: Concepción, Chile.

Granados, J.; Vargas, C.; Vargas, R. (2020). La formación de Profesionales competentes e Innovadores mediante el uso de metodologías activas. Revista Universidad y Sociedad, 12(1), 343-349. 
Hair, J., Black, W., Babin, B., \& Anderson, R. (2014). Multivariate Data Analysis (Seventh Ed). Edinburg Gate: Pearson.

Hernández, I.; Alvarado, J.; Luna, S. (2020). Creatividad e Innovación: competencias genéricas o transversales en la formación profesional. Revista Virtual Universidad Católica del Norte, 44, 135-151. https://revistavirtual.ucn.edu.co/index.php/RevistaUCN/article/view/620/115

Hernández, L.; Ceniceros, D. (2018). Autoeficacia docente y desempeño docente, ¿una relación entre variables?. Innovación educativa, 18(78). http://www.scielo.org.mx/pdf/ie/ v18n78/1665-2673-ie-18-78-171.pdf

Johnson, D.; Johnson, R.; Holubec, E. (1999). El aprendizaje cooperativo en el aula. Buenos aires: Paidós.

Maluenda J. (2019). Innovation Project Based Learning. El método y directrices para su implementación [Internet]. Webpage personal Jorge Maluenda. 2019 Recuperado de: http:// jorgemaluendaa.eshost.com.ar/wp-ontent/uploads/2020/10/Innovation-Project-BasedLearning.-El-\%C3\%A9todo-ydirectrices-para-su-implementaci\%C3\%B3n.pdf

Maluenda, J. y Lledó, P. (2019). ¿Cómo medir el aprendizaje en Innovación? Análisis factorial confirmatorio del Innovator's Behavior Questionnaire (i)BQ en universitarios chilenos. En Arancibia, M. L.; Romero, R. y Maregatti, C. (coords.) Innovación educativa en contextos inclusivos de Educación Superior. Barcelona: Octaedro. https://doi.org/10.36006/16183-06

Maluenda, J.; Dubó, S. (2018). Estrategia metodológica para enseñar Innovación en Estudiantes de cinesiología. Revista de la Fundación Educación Médica, 21(5): 235-237. http://scielo. isciii.es/pdf/fem/v21n5/2014-9832-fem-21-5-235.pdf

Navarro, G.; Vacarezza, G.; González, M.; Catalán, R. (2015). Construcción de conocimiento en educación superior: Educación de competencias genéricas en la Universidad de Concepción, Chile. Concepción: Editorial Universidad de Concepción. http://docencia.udec.cl/unidd/ images/stories/contenido/material apoyo/construccion-de-conocimiento-en-educacionsuperior.pdf

Panadero, E. (2017). A Review of Self-regulated Learning: Six Models and Four Directions for Research. Frontiers in Psychology, 8, 422. https://doi.org/10.3389/fpsyg.2017.00422

Pastor, M.; Rodríguez, P.; Ramos, A. (2017). Efectos del financiamiento público a la Innovación: Perspectiva microeconómica a partir de un estudio en pequeñas Empresas. Región y Sociedad, 29 (70). https://doi.org/10.22198/rys.2017.70.a346

Sebastian J. (2010). La Innovación, entre la ciencia, la ficción y la política. Revista Iberoamericana de Ciencia, Tecnología y Sociedad, 2, 3-19.

Tarango, J. ; Guajardo-Morales, I.; Machin-Mastromatteo, J.; Villanueva-Ledezma, A. (2020). Gusto y disposición por la Innovación científico-tecnológica en Estudiantes mexicanos de educación media superior. Información Tecnológica, 31(1), 91-102. http://dx.doi.org/10.4067/S0718$\underline{07642020000100091}$

Vizcaino, A.; López, K.; Klimenko, O. (2018). Creencias de autoeficacia y desempeño docente de profesores universitarios. Katharsis, 25, 75-93. http://revistas.iue.edu.co/index.php/ katharsis7

Zimmerman, B. J.; Labuhn, A. S. (2012). Self-regulation of Learning: Process Approaches to Personal Development. In APA Educational Psychology Handbook: Theories, Constructs, and Critical Issues, 1era edición, 399-425. Washington: American Psychological Association. 